\title{
New principles of cancer therapy give new hope for oncological patients
}

Nobel Prize in Physiology and Medicine 2018

\author{
Bernadic M Jr${ }^{1}$, Duchon $\mathrm{R}^{1}$, Aziri $\mathrm{R}^{1}$, Mladosievicova $\mathrm{B}^{2}$ \\ Department of Surgical Oncology of National Cancer Institute and Slovak Medical University, \\ Bratislava, Slovakia. bernadic.m@gmail.com
}

\begin{abstract}
The Nobel Prize in Physiology or Medicine 2018 was awarded jointly to James P. Allison and Tasuku Honjo „for their discovery of cancer therapy by inhibition of negative immune regulation“. A number of therapeutic approaches are available for cancer treatment, including surgery, radiation, and other strategies, some of which have been awarded previous Nobel Prizes. These include methods for hormone treatment for prostate cancer (Huggins, 1966), chemotherapy (Elion and Hitchins, 1988), and bone marrow transplantation for leukemia (Thomas, 1990). Many scientists engaged in intense basic research and uncovered fundamental mechanisms regulating immunity and also showed how the immune system can recognize cancer cells. T-cells were shown to have receptors that bind to structures recognized as non-self and such interactions trigger the immune system to engage in defense. However, additional proteins acting as T-cell accelerators are also required to trigger a full-blown immune response. Many scientists contributed to this important basic research and identified other proteins that function as brakes on the T-cells, inhibiting immune activation. This intricate balance between the accelerators and inhibitors is essential for a tight control. New strategy was developed into a therapy for humans. Promising results soon emerged from several groups, and in 2010 an important clinical study showed striking effects in patients with advanced melanoma. In several patients, signs of remaining cancer disappeared. The results were dramatic, leading to long-term remission and possible cure in several patients with metastatic cancer, a condition that had previously been considered essentially untreatable. Such remarkable results had never been seen in this patient group before (Fig. 2, Ref. 12). Text in PDF www.elis.sk.

KEY WORDS: Nobel Prize, physiology, medicine, immune system, inhibition, cancer therapy.
\end{abstract}

Every year, all scientific world observes the work of 50 Nobel Prize committee members at Karolinska University in Stockholm with a great interest, especially the physicians observe the awards of the Nobel Prize in Physiology and Medicine. For several years, the Nobel Prize was expected for discoveries in oncology, carcinogenesis, mechanisms of development of oncological diseases, participation of the immune system in progression of oncological disease, treatment options. Our expectation was confirmed - The Nobel Prize in Physiology and Medicine was awarded to two scientists: Allison and Honjo for their discovery of a new principle of cancer treatment, the inhibition of negative immune regulation (Fig. 1).

Cancer is the leading cause of morbidity and mortality in the most developed countries in the world. The highest occurrence of colorectal carcinoma in the world is in Slovakia. The occurrence of prostate, cervical and breast cancer is also alarming... The cor-

${ }^{1}$ Department of Surgical Oncology of National Cancer Institute and Slovak Medical University, Bratislava, Slovakia, and ${ }^{2}$ Department of Pathophysiology, School of Medicine, Comenius University, Bratislava, Slovakia.

Address for correspondence: M. Bernadic, MD, PhD, Department of Surgical Oncology of National Cancer Institute and Slovak Medical University, Klenova 1, SK-833 10 Bratislava, Slovakia.

Phone: +420.903846184 relation between the immune system and the formation of various types of cancer is apparent for a long time. The Nobel Prize laureates were focused on the functions of the immune system.
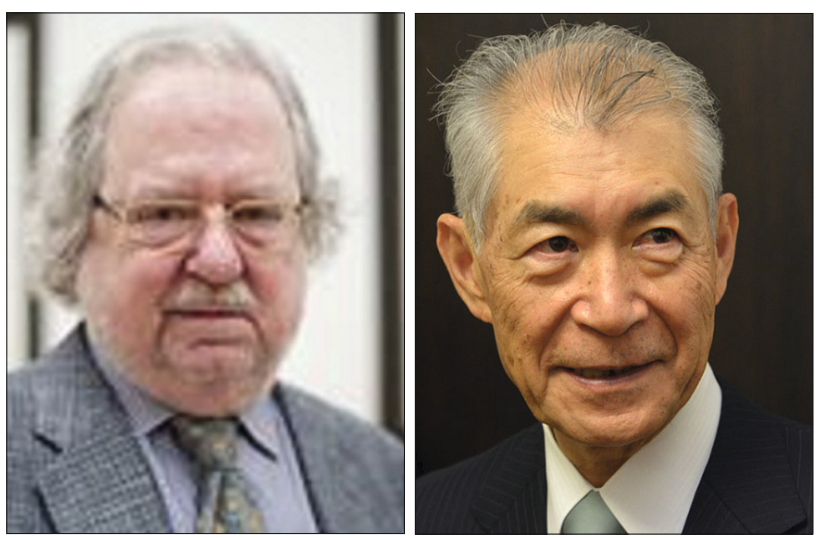

Fig. 1. James P. Allison and Tasuku Honjo win Nobel Prize for Physiology and Medicine 2018. Professor J.P. Allison was born in Alison (Texas), from the 2012nd he works at Anderson Cancer Center University of Texas in Houston and Parker's Institute of cancer immunotherapy. Professor Tasuku Honjo was born at Kyoto (Japan), from the 1984th is professor at Kyoto University, where was dean of Medical School, too (1996-2000; 2002-2004). 


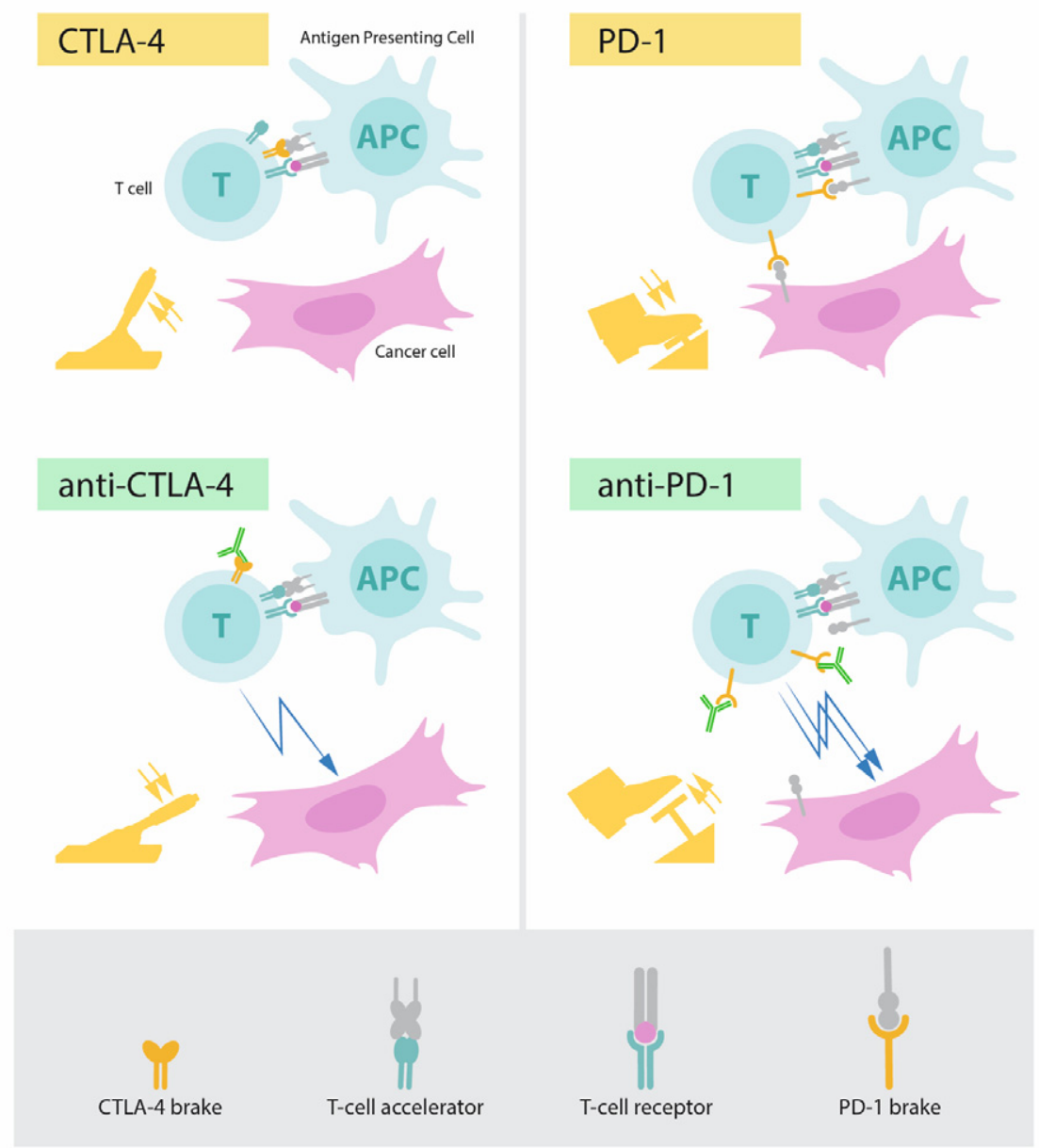

Fig. 2. Upper left: Activation of T cells requires that the T-cell receptor binds to structures on other immune cells recognized as "non-self”. A protein functioning as a T-cell accelerator is also required for $T$ cell activation. CTLA- 4 functions as a brake on T cells that inhibits the function of the accelerator. Lower left: Antibodies (green) against CTLA-4 block the function of the brake leading to activation of T cells and attack on cancer cells. Upper right: PD-1 is another T-cell brake that inhibits T-cell activation. Lower right: Antibodies against PD-1 inhibit the function of the brake leading to activation of $T$ cells and highly efficient attack on cancer cells.

Allison described a regulatory protein found on the immune cells, which is known to act as a breaking mechanism for the immune system $(1,2)$. He recognized, that by easing off this brake, the function of the immune system unblocks and (accelerated, maybe uncontrolled) immune reaction could attack cancer cells as well. This way Allison discovered a new principle in cancer treatment.

Meanwhile, Honjo identified a different regulating protein in immune cells, which acts as a brake as well, however functions differently as the one discovered by Allison. Honja's research led to the possibility of using this mechanism for an effective cancer therapy as well (3).

Gaining an insight into regulating mechanisms of natural immunity and their utilization in cancer treatment can be considered the biggest contribution of both Nobel Prize laureates. Cancer is not one disease. It has many causes and the etiopathogenesis of various processes differs significantly. Common sign is an uncontrolled expansion of altered cells, which have a high potential for division and expansion into neighbourhood, to nearby and distant tissues and organs. It is logical that the methods of the treatment will be different according to the different etiopathogenesis - from chemotherapy and radiation exposure to chirurgical elimination of the cancer tissues. Vaccination (cervical cancer), preventive medical examination, non-invasive examination (breast cancer), monitoring of the various cancer markers (prostate cancer and others) are nowadays used in the prevention of several types of cancer. Identification of the primary tumour as well as distant metastases and their target therapy remains a problem in the advanced disease. Various authors were awarded the Nobel Prize for prominent discoveries in oncology. For example: hormonal treatment of prostate cancer (Huggins, 1966), chemotherapy (Elion, 1988) and bone marrow transplantation during leukaemia (Thomas, 1990). It has to be mentioned that carcinogenesis research denotes the most considerable discoveries. In spite of knowledge advances, oncological diseases are still considered the most serious ones. Cancer in advanced stage remains practically incurable. 
Nowadays, the whole problem is focused on the research of the regulatory functions of the immune system. From the description of the immune system as such at the end of 19th and beginning of the 20th century, the most considerable advances appear in the etiopathogenesis of oncological diseases. It is known, that altered cells (damaged, or dying) are identified, marked and destroyed by the immune system. A certain level of the immune system failure therefore participates at the start of the neoplastic process. Many scientists were focused on the research of regulatory mechanisms of immune processes. It can be said, that in recent years this research made a significant advance by discovering the so called regulatory proteins. The Nobel Prize for discoveries in this area was expected for several years. Yet the results of the basic research of James P. Allison and Tasuka Honja moved forward several open questions into an implementation outcome for the oncological patients.

The primary assumption of the right immune system functionality is the ability to distinguish own and foreign tissues and cells. All foreign cells (bacteria, viruses, damaged or altered cells) must be identified and destroyed. T-lymphocytes are the key players in this defence. It is known that T-lymphocytes have receptors, which bind foreign antigens and activate immune system in order to start a defence reaction.

The system of the immune system function distinguishes another regulatory features, e.g. accelerators and inhibitors. Regulatory proteins can inhibit the function of the immune system, thus preventing the engagement of the immune system, or, on the other hand, accelerate it (Fig. 2).

This system is in complicated equilibrium, which assures, that the immune system is equally engaged in the defence against foreign cells and tissues, but simultaneously, its excess activity, which could lead to autoimmune destruction of the own cells and tissues, is prevented. New knowledge about the function of the immune system was regularly strived to be used by scientists in therapy. In 1990, James P. Allison studied T-lymphocytes and CTLA-4 protein at Karolinska University (Berkeley). He was one of those, who found out that CTLA-4 functions as a brake for T-lymphocytes. Other research teams used this mechanisms in therapy of autoimmune disease (they used inhibitory effect on the process of the autoimmune destruction). However, Allison elaborated a different approach - he developed antibodies that would bind CTLA-4 (Fig. 1), thus releasing the immune system to defend the cancer cells. Allison and his colleagues performed the first experiment and the end of 1994. The results were excellent. They successfully cured mice with cancer by eliminating the brake, thus unleashing the activity of the T-lymphocytes. It is surprising, that pharmaceutical industry had small interest in this breakthrough. Despite, Allison continued in the effort in developing an effective treatment for oncological patients based on this principle. Promising results appeared in several experimental groups. In 2010, an important clinical study proved even unexpected great results in the group of patients with an advanced skin melanoma. In several patients, the cancer was completely cured. These results were never before accomplished in this group of patients.
Regulatory protein PD-1 and its importance in cancer treatment was described by Tasaku Honjo already in $1992(4,5)$, hence several before Allison. T. Honjo described the function of PD-1 in the series of elegant experiments, which he made in the course of several years it the laboratory at Kyoto University. The results showed, that PD-1 as well as CTLA-4, acts as a break for the activity of T-cells. It seems that the same effect on T-lymphocytes can be accomplished by various ways using different types of regulatory proteins. Experiments on animals showed very promising results in inhibiting PD-1 in cancer treatment as well. Verification of the results in clinical studies in 2012 proved a high effectivity in the treatment of patients with various types of cancer (6). Also these results were dramatic, the treatment led to a long-term remission. Several patients with metastasis were successfully cured, which is considered incurable! Likewise in other types of cancer treatment, adverse effects can be severe, eventually life threatening as well. They are caused by hyperactivity of the unblocked immune system, which can lead to autoimmune self-destructing reactions. These adverse effects could be managed as well. However, these situations are studied intensively in order to improve the treatment and decrease the incidence of the adverse effects.

Among these two approaches to treatment, the usage of PD-1 showed to be more effective, for example in lung and renal cancer, lymphoma and melanoma. New clinical studies suggest that the combination of this treatment - focused on CTLA-4 and PD-1 could be even more effective, as was shown in the patients with melanoma.

\section{Conclusion}

It can be summarised that Allison and Honjo discovered a new way, which allows to use the activity of the immune system in the cancer treatment $(8,11)$. With targeted-therapeutic intervention into the immune system equilibrium - through inhibition or activation - it is possible to guide the immune system to eliminate malignant transformed cells. Nowadays, a whole number of controlled clinical studies is ongoing in prestigious centres for the purpose to open new possibilities of treatment in the majority of cancer types. The Nobel Prize in medicine is for both scientists a well-deserved reward for the new hope they have provided to humankind.

A new area opens also in the evaluation of various cancer markers, which nowadays don't have an informative value. Majority of them are carcinoma antigens, which can be under certain circumstances detected and eliminated by the immune system ( 9 , 10). Another concern is, whether these carcinoma antigens have the functions of the regulatory proteins, which enter the complicated equilibrium of activating and inhibiting the immune system and whether they contribute to the progression and spreading of the malignant process. There is a lot of some immunomodulatory proteins and its relations to progression of cancer (12). When these relations are established, a new pathway is open to diagnosis and certainly also to cure many types of oncological diseases. 


\section{5-18}

\section{References}

1. Leach DR, Krummel MF, Allison JP. Enhancement of antitumor immunity by CTLA-4 blockade. Science 1996; 271 (5256): 1734-1736.

2. Kwon ED, Hurwitz AA, Foster BA et al. Manipulation of T cell costimulatory and inhibitory signals for immunotherapy of prostate cancer. Proc Natl Acad Sci USA 1997; 94 (15): 8099-8103.

3. Chowdhury PS, Chamoto K, Honjo T. Combination therapy strategies for improving PD-1 blockade efficacy: a new era in cancer immunotherapy. J Intern Med 2018; 283: 110-120.

4. Ishida Y, Agata Y, Shibahara K, Honjo T. Induced expression of PD-1, a novel member of the immunoglobulin gene superfamily, upon programmed cell death. EMBO J 1992; 11 (11): 3887-3895.

5. Nishimura H, Nose M, Hiai H, Minato N, Honjo T. Development of Lupus-like Autoimmune Diseases by Disruption of the PD-1 gene encoding an ITIM motif-carrying immunoreceptor. Immunity 1999; 11: 141-151.

6. Iwai Y, Terawaki S, Honjo T. PD-1 blockade inhibits hematogenous spread of poorly immunogenic tumor cells by enhanced recruitment of effector T cells. Int Immunol 2005; 17 (2): 133-144.
7. Hodi FS, Mihm MC, Soiffer RJ et al. Biologic activity of cytotoxic T lymphocyte-associated antigen 4 antibody blockade in previously vaccinated metastatic melanoma and ovarian carcinoma patients. Proc Natl Acad Sci USA 2003; 100 (8): 4712-4717.

8. Freeman GJ, Long AJ, Iwai $Y$ et al. Engagement of the PD-1 immunoinhibitory receptor by a novel B7 family member leads to negative regulation of lymphocyte activation. J Exp Med 2000; 192 (7): 1027-1034.

9. Kaušitz J et al. Nádorové markery. Bratislava: Solen, 2014, 152 pp.

10. Rovenský J et al. Systémový lupus erythematosus. Bratislava: SAP, 2013, 444 pp.

11. Peggs KS, Quezada SA, Allison JP. Cancer immunotherapy: costimulatory agonists and co-inhibitory antagonists. Clin Exp Immunol 2009; 157: 9-19.

12. Pitekova B, Uhlikova E, Kupcova V, Durfinova M, Mojto V, Turecky L. Can alpha-1-acid glycoprotein affect the outcome of treatment in a cancer patient? Bratisl Med J 2019; 120 (1): 9-14.

Received November 7, 2018. Accepted November 29, 2018. 